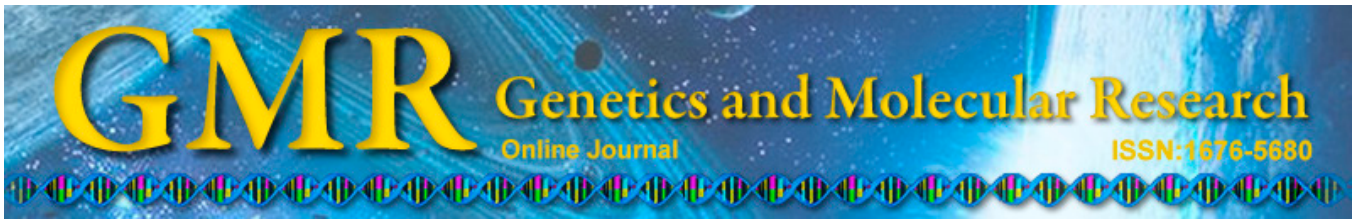

\title{
Identification of molecular markers in patients with hypertensive heart disease accompanied with coronary artery disease
}

\author{
H. Pang, B. Han, Z.Y. Li and Q. Fu \\ Department of Cardiovascular Medicine, Central Hospital of Xuzhou, \\ Xuzhou Clinical School of Xuzhou Medical College, \\ Affiliated Hospital of Southeast University, Xuzhou, China \\ Corresponding author: Q. Fu \\ E-mail: fuqiang_2013@126.com
}

Genet. Mol. Res. 14 (1): 93-100 (2015)

Received March 2, 2014

Accepted October 28, 2014

Published January 15, 2015

DOI http://dx.doi.org/10.4238/2015.January.15.12

\begin{abstract}
We investigated the plasma hypersensitive C-reactive protein (hs-CRP), oxidized low-density lipoprotein (ox-LDL), and apelin levels in patients with hypertensive heart disease (HHD) plus coronary artery disease (CAD). Patients with hypertension hospitalized in Xuzhou Central Hospital were categorized into the HHD group and the HHD plus CAD group; 40 healthy subjects served as the control group. hs-CRP expression was determined with chemiluminescence. The expression of ox-LDL and apelin was analyzed with enzymelinked immunosorbent assay. HHD was chiefly responsible for left atrial enlargement $(\mathrm{P}<0.05)$ and left ventricle diastolic function insufficiency $(\mathrm{P}<0.05)$. hs-CRP and ox-LDL were significantly higher in the HHD group than in the control group $(\mathrm{P}<0.05)$. Compared with the control and HHD groups, significant increases in hs-CRP and ox-LDL levels were observed in the HHD plus CAD group. Apelin expression significantly decreased in the HHD group compared with that in controls $(\mathrm{P}<0.05)$. Meanwhile, apelin expression significantly decreased in the HHD plus CAD group compared with that in the
\end{abstract}


HHD $(\mathrm{P}<0.05)$ and control $(\mathrm{P}<0.05)$ groups. Logistic regression analysis for the binomial response variable indicated that high systolic pressure/diastolic pressure, increase in hs-CRP level, and decrease in apelin concentration were the risk factors for hypertension and cardiac impairments. HHD plus CAD has a greater influence on cardiac function than HHD alone. Increased inflammation and oxidative stress, as well as decreased secretion of cardiac protective factors, may be associated with the simultaneous onset of HHD and CAD.

Key words: Hypertensive heart disease; Coronary artery disease; High sensitivity C-reactive protein; Ox-LDL; Apelin

\section{INTRODUCTION}

Hypertension, a chronic medical condition of elevated blood pressure in the arteries, has been considered one of the most frequently reported cardiovascular diseases. Coronary artery disease (CAD), one of the most hazardous complications of hypertension, has caused great threat to public health. Data from population monitoring show that the annual morbidity of CAD events is 50/100,000, with an increasing tendency in recent years (Writing Group of 2010 Chinese Guidelines for the Management of Hypertension, 2011). Long-term increase in blood pressure may lead to changes in heart structure and diastolic/ systolic functions. In patients with hypertension and CAD, cardiac function impairments may deteriorate.

In this study, we aimed to compare the concentrations of plasma hypersensitive Creactive protein (hs-CRP), oxidized low-density lipoprotein (ox-LDL), and apelin levels in patients with hypertensive heart disease (HHD), and those with HHD plus CAD. Accordingly, the impairments in cardiac target organs induced by these factors were investigated from the viewpoints of inflammatory reactions, oxidative stress, and cardiac protective factors. Our study can provide certain theoretical basis for the early prevention and treatment of hypertension plus CAD.

\section{MATERIAL AND METHODS}

Patients with hypertension hospitalized in the Department of Cardiology in $\mathrm{Xu}-$ zhou Central Hospital from April 2012 to September 2012 were enrolled in this study. All patients signed the informed content form. This study was approved by the Ethics Committee of Xuzhou Central Hospital. The inclusion criteria were as follows: patients with a history of hypertension and patients who were finally evaluated as having hypertension during hospitalization in our hospital. The exclusion criteria included the following: patients with secondary hypertension, hypertrophic cardiomyopathy, and dilated cardiomyopathy, and those with myocardial diseases with definite causes. The diagnosis of hypertension was based on the National Guide to Prevention and Treatment of Hypertension in 2010 (Writing Group of 2010 Chinese Guidelines for the Management of Hypertension, 2011): systolic pressure $\geq 140 \mathrm{mmHg}(1 \mathrm{mmHg}=0.133 \mathrm{kPa})$ and/or diastolic pressure $\geq 90 \mathrm{mmHg}$ at rest, or definite medical records for hypertension and current administration with antihypertensive drugs. HHD was diagnosed by combining these find- 
ings with medical records for hypertension, symptoms and physical signs of the patients, electrocardiogram, chest radiograph, and cardiac ultrasound cardiogram. CAD is diagnosed according to the diagnostic criteria of the American Heart Association/American College of Cardiology established in 2008 (Krumholz et al., 2008). Specifically, coronary arteriography should confirm that at least one coronary artery in the three major coronary arteries, i.e., left anterior descending branch, left circumflex coronary artery, and right coronary artery, showed a stenosis with an internal diameter $>50 \%$. Diabetes is diagnosed using the criteria of the European Diabetes Association in 2007 (Ryden et al., 2007): fasting venous blood sugar $\geq 7.0 \mathrm{mM}$ and/or venous blood sugar $\geq 11.1 \mathrm{mM}$ at $2 \mathrm{~h}$ after meals, or definite medical records for diabetes or current administration with hypoglycemic agents.

Before the study, general patient information was collected, including age; gender; smoking; mean systolic pressure/diastolic pressure; complications such as diabetes, hyperlipidemia, atrial fibrillation, CAD, renal impairments, and HHD; as well as drug medication.

The number of diseased coronary arteries was calculated according to the previous description by Melvin Paul Judkins. Briefly, the characteristics of the patients were determined in terms of the location and degree of luminal narrowing, a limitation of the conventional angiographic classification of patients into subgroups of those having single-, double-, and triple-vessel coronary diseases.

The diagnostic parameters for the ultrasound cardiogram of HHD are discussed as follows. The parameters for the changes in cardiac structure included i) left atrial enlargement: anteroposterior diameter of the left auricle $\geq 35 \mathrm{~mm}$; ii) left ventricular enlargement: left ventricle diastasis diameter $\geq 55 \mathrm{~mm}$ in male patients and $\geq 50 \mathrm{~mm}$ in female patients; iii) left ventricular hypertrophy: thickness of the interventricular septum and/or left ventricular posterior wall $\geq 12 \mathrm{~mm}$. The parameters for cardiac function included the following: i) decreased diastolic function of the left ventricle: abnormal mitral ratio of peak early to late diastolic filling velocity (E/A ratio); ii) decreased systolic function of the left ventricle: left ventricular ejection fraction $\leq 45 \%$.

For plasma level detection, blood samples were collected in a fasting state the morning after hospitalization. The hs-CRP content was determined using the kits purchased from Maglumi Co., Ltd. (Shenzhen, China), strictly following manufacturer instructions. The ox-LDL and apelin levels were determined using an enzyme-linked immunosorbent assay according to manufacturer instructions provided by Mercodia Inc. (Uppsala, Sweden) and Phoenix Pharmaceuticals Inc. (Belmont, CA, USA).

All data are reported as means \pm SD. The data were analyzed using the SPSS13.0 statistical software (SPSS Inc., Chicago, IL, USA). The Student $t$-test was carried out for the interclass comparison between two samples, and variance analysis was carried out for the interclass comparison among multiple samples. The qualitative data were represented by total case number and percentage. The chi-square test was carried out for the interclass comparison. Single-factor logistic regression analysis was performed to identify the risk factors for patients with hypertension and complicated impairments in the cardiac target organs. $\mathrm{P}$ $<0.05$ demonstrated statistical difference.

\section{RESULTS}

Table 1 summarizes the patient information. In this study, 1148 hospitalized patients (628 men, 520 women) with hypertension were enrolled. The age of patients for investiga- 
tion was 33 to 93 years (mean, $66.78 \pm 11.42$ years). The median age was 63 years, and the oldest age was 60 years. Interclass comparison indicated that the percentage of left atrial enlargement in the patients with HHD was higher than that of left ventricular enlargement, followed by that of left ventricular hypertrophy $(\mathrm{P}<0.05)$. Meanwhile, the percentage of left ventricle diastolic function insufficiency was higher than that of left ventricle systolic function insufficiency $(\mathrm{P}<0.05)$.

Table 1. Baseline clinical characteristics of patients with hypertension $(\mathrm{N}=1148)$.

\begin{tabular}{lc}
\hline Characteristics & Value \\
\hline Age (years) & $66.78 \pm 11.42$ \\
Gender [N (\%)] & $628(54.70)$ \\
Male & $520(45.30)$ \\
Female & $141.62 \pm 16.91$ \\
SBP (mmHg) & $83.37 \pm 14.31$ \\
DBP (mmHg) & \\
Risk factor [N (\%)] & $200(17.42)$ \\
Smoking & $264(23.00)$ \\
Diabetes & $392(34.15)$ \\
Hyperlipidemia & $144(12.54)$ \\
Atrial fibrillation & $217(18.90)$ \\
Renal impairments & $756(65.85)$ \\
CHD & $252(33.33)$ \\
Single-vessel CAD & $258(34.13)$ \\
Double-vessel CAD & $246(32.54)$ \\
Triple-vessel CAD & $220(19.16)$ \\
HHD & \\
Impairment of cardiac structure [N (\%)] & $164(74.54)$ \\
LAE & $100(45.45)$ \\
LVE & $66(9.00)^{\mathrm{a}}$ \\
LVH & $128(58.18)$ \\
Impairment of cardiac function [N (\%)] & $46(20.91)^{\mathrm{b}}$ \\
Left ventricular diastolic dysfunction & \\
Left ventricular systolic dysfunction & .
\end{tabular}

$\mathrm{SBP}=$ systolic blood pressure; $\mathrm{DBP}=$ diastolic blood pressure CHD $=$ coronary heart disease $; \mathrm{CAD}=$ coronary artery disease; $\mathrm{HHD}=$ hypertensive heart disease; $\mathrm{LAE}=$ left atrial enlargement; $\mathrm{LVE}=$ left ventricular enlargement; $\mathrm{LVH}=$ left ventricular hypertrophy. ${ }^{\mathrm{P}} \mathrm{P}<0.05$ compared with the LAE and LVE groups. ${ }^{\mathrm{b}} \mathrm{P}<0.05$ compared with the left ventricular diastolic dysfunction group.

Patients with hypertension were divided into two groups: those with or without HHD. No statistical difference was noted in age, gender percentage, and systolic pressure/ diastolic pressure between the two groups $(\mathrm{P}>0.05)$. However, the morbidities of diabetes, hyperlipidemia, renal impairments, and CAD showed a significant increase in patients with HHD $(\mathrm{P}<0.05)$. Patients with triple-vessel CAD $(66.27 \%)$ accounted for $>50 \%$, whereas the percentages of patients with single- or double-vessel CAD were relatively lower. Patients with hypertension not complicated with HHD mostly had single- or double-vessel $\mathrm{CAD}$, and the percentage of patients with triple-vessel CAD was very low. The proportion of patients treated with angiotensin-converting enzyme inhibitor/angiotensin II receptor antagonist (ACEI/ARB), antiplatelet drugs, statins, and sugar-reducing therapy was higher in the HHD group than in hypertension patients without HHD $(\mathrm{P}<0.05$; Table 2$)$.

Patients with HHD $(\mathrm{N}=220)$, after excluding those with diabetes, hyperlipidemia, atrial fibrillation, renal impairments, and smoking, were divided into the following groups: HHD $(\mathrm{N}=24)$ and HHD plus CAD $(\mathrm{N}=35)$. Furthermore, 40 healthy individuals evaluated in the physical examination center of our hospital during the same period were included as 
Table 2. Baseline clinical data of patients with hypertension with or without HHD.

\begin{tabular}{|c|c|c|c|}
\hline Characteristics & Without HHD group $(\mathrm{N}=928)$ & With HHD group $(\mathrm{N}=220)$ & P value \\
\hline Age (years) & $66.41 \pm 11.48$ & $68.32 \pm 10.80$ & 0.136 \\
\hline \multicolumn{4}{|l|}{ Gender [N (\%)] } \\
\hline Male & $516(55.60)$ & $112(50.91)$ & 0.209 \\
\hline Female & $412(44.40)$ & $108(49.09)$ & 0.209 \\
\hline SBP (mmHg) & $141.74 \pm 19.57$ & $141.13 \pm 18.67$ & 0.952 \\
\hline DBP (mmHg) & $83.07 \pm 16.20$ & $84.64 \pm 15.65$ & 0.804 \\
\hline \multicolumn{4}{|l|}{ Risk factor [N (\%)] } \\
\hline Smoking & $166(17.89)$ & $34(15.45)$ & 0.392 \\
\hline Diabetes & $194(20.91)$ & $70(31.82)$ & 0.001 \\
\hline Hyperlipidemia & $298(32.11)$ & $94(42.73)$ & 0.003 \\
\hline Atrial fibrillation & $110(11.85)$ & $34(15.45)$ & 0.147 \\
\hline Renal impairments & $165(17.78)$ & $52(23.64)$ & 0.046 \\
\hline CHD & $590(63.58)$ & $166(75.45)$ & 0.001 \\
\hline Single-vessel CAD & $234(39.66)$ & $18(10.84)$ & 0.000 \\
\hline Double-vessel CAD & $220(37.29)$ & $38(22.89)$ & 0.000 \\
\hline Triple-vessel CAD & $136(23.05)$ & $110(66.27)$ & 0.000 \\
\hline \multicolumn{4}{|l|}{ Medications [N (\%)] } \\
\hline Diuretic & $110(11.85)$ & $29(13.18)$ & 0.855 \\
\hline Calcium channel blocker & $443(47.74)$ & $116(52.73)$ & 0.710 \\
\hline $\mathrm{ACEI} / \mathrm{ARB}$ & $470(50.65)$ & $142(64.55)$ & 0.001 \\
\hline$\beta$-blocker & $267(28.77)$ & $53(24.09)$ & 0.726 \\
\hline Antiplatelet agent & $649(69.94)$ & $181(82.27)$ & 0.001 \\
\hline Statins & $607(65.41)$ & $169(76.82)$ & 0.001 \\
\hline Hypoglycemic agent & $167(18.00)$ & $62(28.18)$ & 0.001 \\
\hline
\end{tabular}

$\mathrm{HHD}=$ hypertensive heart disease; $\mathrm{SBP}=$ systolic blood pressure; $\mathrm{DBP}=$ diastolic blood pressure; $\mathrm{CHD}=$ coronary heart disease; $\mathrm{CAD}=$ coronary artery disease; $\mathrm{ACEI} / \mathrm{ARB}=$ angiotensin-converting enzyme inhibitor/angiotensin II receptor antagonist.

the control group. hs-CRP and ox-LDL levels were significantly higher in the HHD group than in the control group $(\mathrm{P}<0.05)$. Compared with the control group and the HHD group, a significant increase in the hs-CRP and ox-LDL levels was observed in the HHD plus CAD group. The expression of apelin showed a significant decrease in the HHD group compared with that in the control group $(\mathrm{P}<0.05)$. Meanwhile, a significant decrease in apelin level was identified in the HHD plus CAD group compared with that in the HHD group $(\mathrm{P}<$ $0.05)$ and control group $(\mathrm{P}<0.05)$. Logistic regression analysis for the binomial response variable showed that relatively high systolic pressure/diastolic pressure, increase in hs-CRP, and decrease in apelin level were all risk factors for hypertension and cardiac impairments (Tables 3 and 4).

Table 3. Cardiovascular biomarkers of the three study groups.

\begin{tabular}{lcrr}
\hline Characteristics & Control $(\mathrm{N}=40)$ & HHD $(\mathrm{N}=24)$ & HHD $+\mathrm{CHD}(\mathrm{N}=35)$ \\
\hline Age (years) & $67.43 \pm 7.43$ & $67.05 \pm 12.51$ & $68.05 \pm 11.47$ \\
Gender [N (\%)] & $19(47.50)$ & $11(45.83)$ & $17(48.57)$ \\
Male & $21(52.50)$ & $13(54.17)$ & $18(51.43)$ \\
Female & $120.89 \pm 21.13$ & $147.16 \pm 25.39^{\mathrm{a}}$ & $141.23 \pm 19.65^{\mathrm{a}}$ \\
SBP $(\mathrm{mmHg})$ & $75.97 \pm 13.97$ & $87.68 \pm 16.62^{\mathrm{a}}$ & $82.73 \pm 14.83^{\mathrm{a}}$ \\
DBP $(\mathrm{mmHg})$ & $329.44 \pm 118.97$ & $4144.32 \pm 1695.57^{\mathrm{a}}$ & $6868.83 \pm 2071.63^{\mathrm{ab}}$ \\
hs-CRP $(\mathrm{ng} / \mathrm{mL})$ & $33.62 \pm 6.23$ & $47.92 \pm 5.56^{\mathrm{a}}$ & $66.97 \pm 7.02^{\mathrm{ab}}$ \\
ox-LDL $(\mathrm{U} / \mathrm{L})$ & $4.40 \pm 0.71$ & $2.19 \pm 0.46^{\mathrm{a}}$ & $1.37 \pm 0.51^{\mathrm{ab}}$ \\
Apelin $(\mathrm{ng} / \mathrm{mL})$ & & & \\
\hline
\end{tabular}

$\mathrm{HHD}=$ hypertensive heart disease $; \mathrm{CHD}=$ coronary heart disease; $\mathrm{SBP}=$ systolic blood pressure; $\mathrm{DBP}=$ diastolic blood pressure; hs-CRP $=$ hypersensitive $\mathrm{C}$-reactive protein; ox-LDL $=$ oxidized low-density lipoprotein. ${ }^{\text {aP }}<0.05$ compared with the control group. ${ }^{b} \mathrm{P}<0.05$ compared with the HHD group. 
Table 4. Single-factor logistic regression analysis for association between variables and HHD.

\begin{tabular}{|c|c|c|c|c|c|c|c|}
\hline \multirow[t]{2}{*}{ Independent variables } & \multirow[t]{2}{*}{$\beta$} & \multirow[t]{2}{*}{ SE } & \multirow[t]{2}{*}{ Wald } & \multirow[t]{2}{*}{$\mathrm{P}$ value } & \multirow[t]{2}{*}{ OR } & \multicolumn{2}{|c|}{$95 \% \mathrm{CI}$} \\
\hline & & & & & & L-limit & U-limit \\
\hline SBP & 0.177 & 0.085 & 4.392 & 0.000 & 1.194 & 1.012 & 1.409 \\
\hline DBP & 0.003 & 0.001 & 15.862 & 0.000 & 1.003 & 1.002 & 1.005 \\
\hline hs-CRP & 0.238 & 0.069 & 9.560 & 0.005 & 1.269 & 1.075 & 1.498 \\
\hline Apelin & -0.566 & 0.157 & 18.395 & 0.000 & 0.569 & 0.435 & 0.745 \\
\hline Constant & -5.646 & 0.723 & 91.442 & 0.000 & 0.020 & & \\
\hline
\end{tabular}

$\mathrm{SBP}=$ systolic blood pressure; $\mathrm{DBP}=$ diastolic blood pressure; hs-CRP $=$ hypersensitive $\mathrm{C}$-reactive protein.

\section{DISCUSSION}

Hypertension is a progressive vascular syndrome characterized by continuous increase in arterial blood pressure. It is one of the major risk factors for cardiovascular diseases and is always complicated with multiple risk factors, impairments in target organs, or clinical diseases (Tsai, 2011). In our study, 1148 patients with hypertension were enrolled. The complications of the patients with hypertension in the present study included diabetes, hyperlipidemia, atrial fibrillation, renal impairments, CAD, and HHD, among which the morbidity of HHD (65.85\%) was significantly higher than that of the other diseases. The impairments of cardiac target organs caused by hypertension were characterized by left ventricular hypertrophy and decreased compliance at the relaxation period, which was induced by the increase in the burden to the heart after hypertension and was always complicated with left atrial enlargement. If the blood pressure is not well controlled in the long term, it may further develop into left ventricular enlargement and become complicated with systolic function inadequacy, which may affect the cardiac ejection functions. Based on these factors, the 1148 patients in the present study were divided into groups of those with hypertension with or without HHD. No statistical difference was noted in age and systolic pressure/diastolic pressure between the two groups. However, the morbidities of diabetes, hyperlipidemia, renal impairments, and CAD significantly increased in patients with hypertension combined with HHD. Patients with triple-vessel CAD (66.27\%) accounted for $>50 \%$, whereas the percentages of patients with single- or double-vessel CAD were relatively lower. The percentages of patients treated with ACEI/ARB, antiplatelet drugs, statins, and sugar-reducing therapy in the group complicated with HHD were higher than those in the group not complicated with HHD.

The results indicated that the risks for cardiovascular events in the group complicated with HHD were significantly higher after comprehensive evaluations for cardiovascular risk factors, impairments of target organs, clinical complications, diabetes, and other aspects even if the blood pressures were at the same level. Many prospective cohort studies found that strong, continuous, and independent positive correlation can be found between blood pressure and $\mathrm{CAD}$, and the risks for CAD began to increase when the systolic pressure was $>115$ $\mathrm{mmHg}$ (Gupta et al., 2012). HHD is always complicated with coronary atherosclerosis and microangiopathy (Manios et al., 2009), which may finally lead to cardiac failure, severe cardiac arrhythmias, and even sudden death. It was postulated from the data of the present study that besides blood pressure, other risk factors for cardiovascular events, including abnormalities in blood fat metabolism, family history of premature cardiovascular diseases, smoking history, abdominal obesity, hyperinsulinism, insulin resistance, impaired sugar tolerance on an empty stomach, hypercoagulable state, endothelial function disorder, decreased vascular compliance, 
and other comprehensive functions (Glasser et al., 2011), were key factors leading to the aggravation of diffusive lesions in coronary arteries. Therefore, we speculated that these patients might benefit from a comprehensive intervention targeting the multiple risk factors.

hs-CRP plays an important role in predicting the risks for cardiovascular events such as acute coronary syndrome, myocardial infarction, apoplexy, and metabolism syndrome (Hamer et al., 2010). After oxidation in vivo, LDL is metabolized to ox-LDL. ox-LDL can damage the integrity of the endothelium, allowing invasion of monocytes, LDL, and other components into the subendothelial layer, which may lead to atherosclerosis (Bzowska et al., 2012). Furthermore, ox-LDL can also inhibit the production of nitric oxide and its biological activities, and induce abnormalities in the vasodilatation functions. The protective functions of apelin have been well defined in hypertension, myocardial hypertrophy, cardiac failure, myocardial ischemia-reperfusion injury (Rastaldo et al., 2011), and other cardiovascular diseases. It can reduce peripheral circulation resistance and regular blood pressure by stretching blood vessels (Iturrioz et al., 2010), prevent arteriosteogenesis (Shan et al., 2011), improve vascular functions, and regulate water-salt balance (Roberts et al., 2009). Additionally, it can also improve cardiac function by reinforcing cardiac contractility (Maguire et al., 2009), limit oxidative stress, and inhibit cardiac muscle cell hypertrophy (Zhang et al., 2009) and apoptosis (Foussal et al., 2010). A recent study on 232 patients with primary hypertension (PrzewlockaKosmala et al., 2011) indicated that the decrease in serum apelin level was closely related to disorders in the systolic/diastolic functions of the heart.

Among the 220 patients with HHD, after ruling out the interferences from diabetes, hyperlipidemia, atrial fibrillation, renal impairments, and smoking factors, the results showed that the hs-CRP and ox-LDL levels in the HHD group were significantly higher than those in the control group. In addition, significant increases were seen in the hs-CRP and ox-LDL levels in the HHD plus CAD group compared with those in the HHD and control groups. The apelin level in the HHD group was significantly lower than that in the control group. The apelin level was significantly lower in the HHD plus CAD group than in the HHD and control groups. Higher systolic pressure/diastolic pressure, upregulation of hs-CRP, and downregulation of apelin were the risk factors for hypertension and complicated cardiac impairments. This indicated that aggravation of inflammatory reactions and oxidative stress, together with the decrease in apelin level, contributed to the onset and progression of HHD. In the presence of CAD as a complication, changes in the levels of the factors mentioned above are further aggravated, which may provide important information for the diagnosis of the disease condition and the clinical outcome.

In conclusion, for the management of patients with cardiovascular diseases, the risk grade should first be evaluated to prevent the incidence of cardiovascular events. Patients with a greater number of risk factors such as diabetes, chronic renal diseases, cerebral apoplexy, arteriopathy, and peripheral arteriopathy should be given more attention (Tsai, 2011). Concerning the treatments of hypertension, measures should be taken to reduce blood pressure, comprehensively evaluate the risk grades, and interfere with the risk factors. Moreover, more attention should be given to protecting target organs such as the heart, brain, and kidney. As the hs-CRP, ox-LDL, and apelin levels are closely related to the pathogenesis of primary hypertension, they can be used in the evaluation of pathogenesis in patients with hypertension. Our study provides a new concept for the prediction and diagnosis of hypertension and early screening of populations at high-risk for hypertensive diseases. 


\section{Conflicts of interest}

The authors declare no conflict of interest.

\section{ACKNOWLEDGMENTS}

Research supported by the Xuzhou Municipal Bureau of Science and Technology (\#XZZD1020) and the Xuzhou Municipal Health Bureau (\#XWJ2011030).

\section{REFERENCES}

Bzowska M, Nogiec A, Skrzeczynska-Moncznik J, Mickowska B, et al. (2012). Oxidized LDLs inhibit TLR-induced IL-10 production by monocytes: a new aspect of pathogen-accelerated atherosclerosis. Inflammation 35: 1567-1584.

Foussal C, Lairez O, Calise D, Pathak A, et al. (2010). Activation of catalase by apelin prevents oxidative stress-linked cardiac hypertrophy. FEBS Lett. 584: 2363-2370.

Glasser SP, Judd S, Basile J, Lackland D, et al. (2011). Prehypertension, racial prevalence and its association with risk factors: Analysis of the REasons for Geographic And Racial Differences in Stroke (REGARDS) study. Am. J. Hypertens. 24: 194-199.

Gupta P, Nagaraju SP, Gupta A and Mandya Chikkalingaiah KB (2012). Prehypertension - time to act. Saudi J. Kidney Dis. Transpl. 23: 223-233.

Hamer M, Chida Y and Stamatakis E (2010). Association of very highly elevated C-reactive protein concentration with cardiovascular events and all-cause mortality. Clin. Chem. 56: 132-135.

Iturrioz X, Alvear-Perez R, De Mota N, Franchet C, et al. (2010). Identification and pharmacological properties of E3393D6, the first nonpeptidic apelin receptor agonist. Faseb J. 24: 1506-1517.

Krumholz HM, Anderson JL, Bachelder BL, Fesmire FM, et al. (2008). ACC/AHA 2008 performance measures for adults with ST-elevation and non-ST-elevation myocardial infarction: a report of the American College of Cardiology/ American Heart Association Task Force on Performance Measures (Writing Committee to Develop Performance Measures for ST-Elevation and Non-ST-Elevation Myocardial Infarction) Developed in Collaboration With the American Academy of Family Physicians and American College of Emergency Physicians Endorsed by the American Association of Cardiovascular and Pulmonary Rehabilitation, Society for Cardiovascular Angiography and Interventions, and Society of Hospital Medicine. J. Am. Coll. Cardiol. 52: 2046-2099.

Maguire JJ, Kleinz MJ, Pitkin SL and Davenport AP (2009). [Pyr1] apelin-13 identified as the predominant apelin isoform in the human heart: vasoactive mechanisms and inotropic action in disease. Hypertension 54: 598-604.

Manios E, Tsivgoulis G, Koroboki E, Stamatelopoulos K, et al. (2009). Impact of prehypertension on common carotid artery intima-media thickness and left ventricular mass. Stroke 40: 1515-1518.

Przewlocka-Kosmala M, Kotwica T, Mysiak A and Kosmala W (2011). Reduced circulating apelin in essential hypertension and its association with cardiac dysfunction. J. Hypertens. 29: 971-979.

Rastaldo R, Cappello S, Folino A and Losano G (2011). Effect of apelin-apelin receptor system in postischaemic myocardial protection: a pharmacological postconditioning tool? Antiox. Redox Signal. 14: 909-922.

Roberts EM, Newson MJ, Pope GR, Landgraf R, et al. (2009). Abnormal fluid homeostasis in apelin receptor knockout mice. J. Endocrinol. 202: 453-462.

Ryden L, Standl E, Bartnik M, Van den Berghe G, et al. (2007). Guidelines on diabetes, pre-diabetes, and cardiovascular diseases: executive summary. The Task Force on Diabetes and Cardiovascular Diseases of the European Society of Cardiology (ESC) and of the European Association for the Study of Diabetes (EASD). Eur. Heart J. 28: 88-136.

Shan PF, Lu Y, Cui RR, Jiang Y, et al. (2011). Apelin attenuates the osteoblastic differentiation of vascular smooth muscle cells. PloS One 6: e17938.

Tsai WC (2011). Treatment options for hypertension in high-risk patients. Vasc. Health Risk Manage. 7: 137-141.

Writing Group of 2010 Chinese Guidelines for the Management of Hypertension (2011). 2010 Chinese guidelines for the management of hypertension. Chin. J. Cardiol. 39: 579-616.

Zhang Z, Yu B and Tao GZ (2009). Apelin protects against cardiomyocyte apoptosis induced by glucose deprivation. Chin. Med. J. 122: 2360-2365. 